Published in IET Intelligent Transport Systems

Received on 2nd December 2009

Revised on 7th February 2010

doi: 10.1049/iet-its.2009.0144

Special Issue - selected papers from the 16th

World Congress on ITS

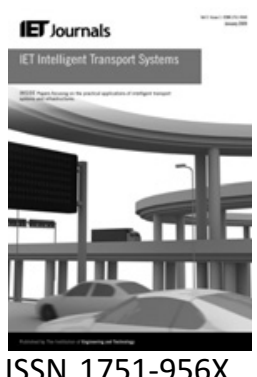

\title{
Auditory signs to support traffic awareness
}

\author{
$\begin{array}{lll}\text { J. Fagerlönn } & \text { H. Alm } \\ & \end{array}$ \\ ${ }^{1}$ Interactive Institute - Sonic Studio, Piteå SE-94128, Sweden \\ ${ }^{2}$ Department of Human Work Science, Luleå University of Technology, Luleå SE-94187, Sweden \\ E-mail: johanf@tii.se
}

\begin{abstract}
In-vehicle information systems (IVIS) may contribute to increased levels of cognitive workload, which in turn can lead to a more dangerous driving behaviour. An experiment was conducted to examine the use of auditory signs to support drivers' traffic situation awareness. Eighteen experienced truck drivers identified traffic situations based on information conveyed by brief sounds. Aspects of learning, cognitive demand and pleasantness were monitored and rated by the drivers. Differences in cognitive effort was estimated using a dual-task set-up, in which drivers responded to auditory signs while simultaneously performing a simulated driving task. As expected, arbitrary sounds required significantly longer learning times compared to sounds that have a natural meaning in the driving context. The arbitrary sounds also resulted in a significant degradation in response performance, even after the drivers got a chance to learn the sounds. Finally, the results indicate that the use of arbitrary sounds can negatively impact driver satisfaction. These results have implications for a broad range of developing intelligent transport systems designed to assist drivers in absence of fundamental visual information or in visually demanding traffic situations.
\end{abstract}

\section{Introduction}

Technical development of intelligent transport systems (ITS) is often associated with expectations of their potential to increase traffic safety [1]. But systems designed to give information could potentially disrupt the driver's ability to maintain full attention on the driving task. This in turn may lead to a more dangerous driving behaviour [2-4] and increase the risk of traffic accidents [5]. Additional tasks might be especially problematic in urgent, unusual and complex situations that already put high demands on the driver's limited attentional resources.

Presenting information visually may not be optimal. Researchers have reported that increased visual load can have negative effects on both detection performance [6] and lane keeping [3]. Thus, interfaces that allow the driver to keep their eyes on the road, such as combined visual and auditory solutions, can be more appropriate from a safety point of view. But research has also demonstrated that involvement in non-visual tasks can have a negative impact on safety. Engström et al. [3] found that an auditory continuous memory task resulted in increased gaze concentration towards the road centre. Numerous studies have reported negative effects of mobile phone conversation on workload and attention $[2,4,7]$.

However, being involved in a verbal conversation is not the same as listening to sounds. McCarley et al. [8] concluded that the negative impact on visual search seen during natural telephone conversation was most likely due to speech reproduction. On the other hand, Richard et al. [9] reported that cognitively demanding auditory messages (that did not require the driver to respond verbally) could affect voluntary visual scanning, and the ability to detect changes in the traffic scene. Thus, there are reasons to investigate the relative potential in auditory messages to raise workload during driving.

In a collaboration project between Scania CV AB, Interactive Institute and Luleå University of technology in Sweden, research addresses how audio design can meet the requirements of safe within-vehicle communication in heavy vehicles. The work presented in this article focus on the effectiveness of auditory signs to amplify traffic situation awareness (SA).

\subsection{Auditory displays to support traffic SA}

The importance of SA for safe driving has been described in the literature [10]. According to Endsley [11] SA is 'the 
perception of the elements in the environment within a volume of time and space, the comprehension of their meaning, and the projection of their status in the near future'. Traffic SA can be seen as one component of the more general construct of SA. Cues that strengthen traffic SA may be regarding other road users and dangers, their position in relation to the own vehicle and how the situation is evolving over time.

One strategy to heighten awareness using auditory signals can be to direct visual attention to important events [12-14]. Fung et al. [12] demonstrated that an effective sound for a forward collision warning system was a simple tone of $2 \mathrm{kHz}$. The sound quickly made drivers pay attention to the road ahead and break fast. In a similar way, attention grabbing visual or tactile cues can be used to make the driver shift visual attention towards crucial information. In a recent study, Ho et al. [14] found that tactile cues may even be more effective that sound for this purpose.

But even though attention-grabbing signals are effective for some urgent events, there are reasons why to examine the use of more complex sound to convey traffic related information. First, many types of ITS such as night vision, blind spot detection and various road user protection systems are introduced in vehicles to assist in conditions and areas with low or no visibility. Other systems are developed to make drivers aware of accident-prone areas and potential dangers, such as intersections, bus stops and school areas. Information through sound can be an attractive means of communication in absence of fundamental visual information. Further, sounds that not only catch attention, but also carry the relevant information, may reduce the risk of visual overload in visually demanding traffic situations.

\subsection{Auditory signs}

A body of research has demonstrated how both verbal and nonverbal auditory signs can be used to convey information in user environments [15-18]. Evaluations of non-verbal signs have been partly focusing on advantages and disadvantages of sound types commonly referred to as earcons and auditory icons. The concept of earcons was first introduced by Blattner et al. [19], who defined them as 'non-verbal audio messages used in the user-computer interface to provide information to the user about some computer object, operation or interaction'. Blattner et al. suggested that earcons, like visual icons, could be divided into following classes: representational, abstract and semi-abstract. Gaver [20] investigated representational earcons, although he referred to them as auditory icons. Gaver defined auditory icons as 'everyday sounds mapped to computer events by analogy with everyday sound producing events'.

Ever since first introduced, earcons, auditory icons and other sound types such as spearcons [17] have been described and evaluated in research [21-26]. Brewster et al. [27] have done some important work evaluating earcons and suggesting design guidelines. They clearly focused on 'hierarchal earcons' and defined them as 'abstract, synthetic tones that can be used in structured combinations to represent parts of an interface'. Auditory icons have been described as an alternative to earcons in that they are nonmusical [17], real-life [22], natural and every-day sounds [21].

At the International Conference for Auditory Display 2008, Mustonen [28] argued that the current definitions of non-verbal auditory signs are not compatible with the sign descriptions of, for example, semiotic science. He stated that 'most signs we encounter are neither purely abstract nor iconic but combine both iconic and symbolic dimensions to make sense'. Further, he pointed out that the same sound could be listened to with different outcomes in different situations and orientations. When listening to interface elements we intuitively recognise familiar parts from the sound and construct the meaning from their relation to the situation'.

Ulfvengren [18] investigated auditory warnings in aviation from a human error perspective. She argued that alerts should be meaningful in the context they are presented. These meaningful sounds are typically cues that exist naturally in the user environment, either as synthetic or non-synthetic sounds. One important aspect in warning design is to find signals that are easily associated to their assigned alert function meaning. Ulfvengren stated that 'If a sound is possible to associate to a given alert function it requires fewer cognitive resources and is therefore appropriate, in this aspect, for auditory alert design'.

\subsection{Auditory signs and traffic $S A$}

McKeown [15] evaluated four sound types (auditory icons, environmental sounds, earcons and speech) to convey various types of in-vehicle information, including some trafficrelated information. Response time, accuracy of response, perceived urgency and scores of pleasantness were evaluated. The results clearly illustrated the potential in using sounds that have a meaning in the driving context. The environmental category of sounds consisted of real-world sounds that were likely to be familiar to drivers, but did not have any specific meaning within the vehicle interface. These sounds resulted in a degraded judgment performance compared to the more driving related sounds that McKeown categorised as auditory icons. It should, however, be pointed out that the response task used in the experiment was not accompanied by a concurrent driving task.

Vilimek and Hempel [21] investigated different sound types (auditory icons, earcons, keywords and long speech messages) to convey non-critical information in vehicles. Affects on short-term memory and choice reaction performance were collected. Earcons resulted in degraded response times compared to other sound types, while the long speech messages had a negative impact on serial recall. As in the study by McKeown, this experiment did not 
include a concurrent driving task. Also, the study focused on information related to vehicle functions, and no information about traffic events was included.

Chen and Jarlengrip [29] evaluated the use of a 3D sound reproduction technique to improve traffic $\mathrm{SA}$ in a number of traffic situations. This study was conducted in a driving simulator and focused on driver acceptance. The authors concluded that auditory icons are 'suitable to this application due to their intuitiveness, distinguishability and relatively low degree of disturbance'.

Auditory icons seem to be more appropriate sounds to convey in-vehicle information than other sound types that has been evaluated in research, at least from a human information processing perspective. This is however not surprising. Mustonen [28], for example, wrote 'the important difference of the earcon paradigm is that the design in auditory icon paradigm has been more focused on how the sound itself, through similarities and metaphors motivates the meaning creation process'. Auditory icons often sound like what they represent. This tends to make them meaningful to users in the specific context they are presented.

\subsection{Objectives}

Is context-specific meaning an important aspect to consider when designing auditory signals for traffic SA? The purpose of the study was to examine differences in learnability, cognitive demand and pleasantness for brief sounds that have a natural meaning in a driving context, and sounds that have been arbitrary mapped to traffic information. Prior to the experiment it was predicted that sounds that have a meaning in the driving context would be easier to learn compared to arbitrary sounds. The primary aim of the experiment was to investigate differences in cognitive effort after the drivers got a chance to learn the meaning of sounds. Differences in cognitive effort were estimated using a dual-task set-up, in which drivers responded to auditory signs while simultaneously performing a simulated driving task. Another aim of the study was to examine how the use of arbitrary sounds can impact driver satisfaction.

\section{Method}

\subsection{Subjects}

Eighteen truck drivers (17 males and 1 female) with selfreported normal hearing participated in the study. Their ages ranged between 22 and 61 (mean 39). Their truck driving experience ranged between 2 and 32 years (mean 16.8) and self-reported annual driving ranged between 2000 and $150000 \mathrm{~km}$ (mean $75410 \mathrm{~km}$ ).

\subsection{Apparatus}

An illustration of the experimental setting is presented in Fig. 1. The experiment was conducted in a Scania R truck

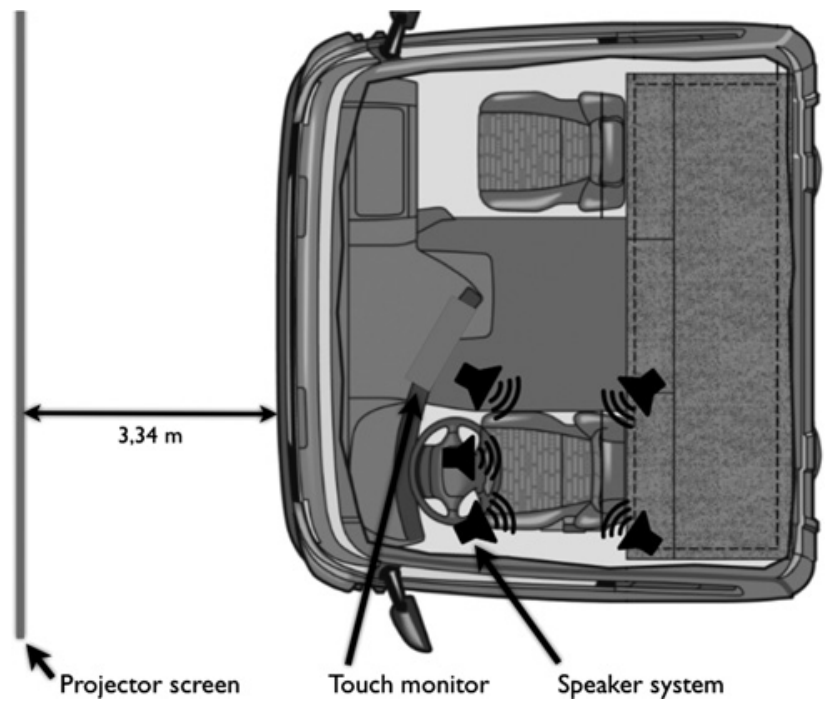

Figure 1 Experimental set-up

cab. A 10,4' touch monitor (Lilliput Electronics, CA, USA) showing videos of four hazardous traffic situations simultaneously during the driving sessions was positioned approximately on one arm length $30^{\circ}$ to the right of the driver. The video clips were brief .gif animations (1.26 s in length, continuously repeating), showing traffic situations from above.

A lane change test represented the driving task. The visual driving scene was projected using an Optoma EP 755 XGA DLP projector (Optoma Technology Inc., CA, USA) projecting an image $3.34 \mathrm{~m}$ in front of the cabin.

Presentation of auditory stimuli, traffic situations and the monitoring of driver responses were handled by a Java application running on a Lenovo Thinkpad T60 (Lenovo, NC, USA). Sound files were processed using a Kontakt 3 sampler (Native Instruments, Berlin, Germany) and an E-MU 1616m digital sound card (E-MU Systems Inc., CA, USA). The sounds were presented to participants at a comfortable listening level through an Anthony Gallo Nucleus Micro 5.1 channel speaker system (Anthony Gallo Acoustics Inc., CA, USA).

\subsection{Dependent and independent variables}

Two sets of non-verbal auditory signs were designed prior to the experiment. Each set contained five sounds mapped to road users (car, truck, pedestrian, children and bicycle). The first set of non-verbal auditory signs (arbitrary) consisted of five short musical motives. Brewster et al. [27] has suggested how musical timbre and rhythm can make sounds distinguishable from each other. Different rhythms and timbres were selected to make the sounds easy to tell apart. The second set of non-speech auditory signs (meaningful) consisted of sounds that are assumed to be 
Table 1 Description of auditory signs used in the experiment

\begin{tabular}{|l|c|c|c|}
\hline & Meaningful & Arbitrary & Verbal \\
\hline bicycle & bicycle bell & piano/G3-C3 - G3-C3 & 'bicycle' \\
\hline car & car horn & snare drum/roll & 'car' \\
\hline truck & truck horn & cello/C\#2-D\#2-F2-G\#2 - C\#2-D\#2-F2-G\#2 & 'truck' \\
\hline pedestrian & man shout & marimba/D3-A3-A3-A3-A3-A3-A3 & 'pedestrian' \\
\hline children & children laugh & trumpet/G4-F\#4-F4-E4-E4-E4 & 'children' \\
\hline
\end{tabular}

meaningful to drivers. Road users make noise and they have their natural ways of catching the attention of other road users. These naturally occurring sounds should typically be meaningful to experienced drivers.

The final non-verbal signs are presented in Table 1. A set of speech messages was also designed and included in the experiment. A soft-spoken male voice was used for the verbal signs (verbal). Each speech message consisted of a keyword presented in Swedish describing the road danger. The different auditory signs were not exactly equal in length but ranged between 1 and $2 \mathrm{~s}$. Spatial positions of road users were represented by presenting the sounds in the corresponding direction (front, rear, front-left, front-right, rear-left and rear-right). Combinations of road user types and positions resulted in a total number of 30 different traffic situations used in the study.

Learning time and trials, response time in judging traffic situations, accuracy of response (identity and position of road dangers) and subjective ratings of cognitive effort and pleasantness defined the dependent variables.

\subsection{Procedure}

The experiment was conducted using a within-subjects design. The participants were introduced to the simulator and the lane change test in a 5-10 min test drive. The experimenter also provided a short demonstration session explaining the judgment task. Subjects were told that they were evaluated on the basis of driving performance. They were required to judge the auditory signals as fast as possible so that it would not affect the driving more than absolutely necessary. However, no parameters related to driving performance were actually monitored during the trials.

The trial consisted of three blocks, one for each condition, which lasted for about $30 \mathrm{~min}$ each. Every block started with a learning session, without the driving task, in which the participants were required to learn the intended mappings between sounds and road users. Images of the road users were presented on the touch screen and the driver were required to play the sounds until he/she felt comfortable with their meaning. Both learning time and trials were monitored.
The learning session was followed by the driving session. This part started with about $5 \mathrm{~min}$ of driving training without sounds. The driving task lasted for about $25 \mathrm{~min}$. The auditory signs were presented in random order to the drivers with $20-60 \mathrm{~s}$ intervals. When a sound was played the drivers were required to select one out of four traffic situations presented on the touch screen. After each judgment the driver received feedback about their response. A green light indicated a correct response. A red light and text presenting the correct answer indicated an incorrect response. This feedback was built-in to allow drivers to learn from mistakes during the sessions. Directly after each driving session, the drivers rated the perceived cognitive effort and pleasantness using rating scales presented on the touch screen. These scales ranged from 1 (not at all challenging/annoying) to 10 (very challenging/annoying).

A loosely structured interview was conducted at the end of each trial in order to obtain complementary driver judgments. In this interview, the drivers were allowed to talk freely about any issues experienced during the trials. The experimenter especially paid attention to the participants' personal experiences regarding how they constructed meaning from the sounds.

\section{Results}

The statistical analysis of the data was performed using the computer package Minitab (Minitab Data Analysis Software, Philadelphia, PA, USA).

\subsection{Learning}

Average learning times and number of trials for the sign sets is presented in Table 2 . As predicted, the drivers had serious problems learning the mappings between the arbitrary sounds and the traffic events. That is, the drivers listened to each sound 5.9 times compared to 1.93 times for the set of meaningful non-verbal signs and 1.52 times for the verbal signs. A one-way analysis of variance (ANOVA) revealed significant differences in learning time $F(2,34)=21.03$, $p<0.01$ and trials $F(2,34)=28.22, p<0.01$. Post-hoc analyses were conducted using Tukey's honestly significantly different (HSD) test. Both learning time and trials were significantly higher in the arbitrary condition than the two other conditions ( $p=0.01)$. 
Table 2 Mean learning time and number of trials

\begin{tabular}{|l|l|l|}
\hline & Time in seconds (SD) & Trials (SD) \\
\hline meaningful & $24.7(8.7)$ & $9.6(2.9)$ \\
\hline arbitrary & $90.2(59.6)$ & $29.5(16.4)$ \\
\hline verbal & $23.3(12.4)$ & $7.6(3.3)$ \\
\hline
\end{tabular}

Table 3 Mean response time and accuracy of response

\begin{tabular}{|l|l|l|}
\hline & Time in seconds (SD) & Error \% (SD) \\
\hline meaningful & $3.00(0.67)$ & $13.7(10.5)$ \\
\hline arbitrary & $4.12(1.30)$ & $33.0(14.6)$ \\
\hline verbal & $3.11(0.62)$ & $12.2(9.0)$ \\
\hline
\end{tabular}

\subsection{Cognitive effort}

Table 3 shows response times and response accuracy for the three conditions and Table 4 presents response times, accuracy of responses in terms of identity of road dangers and position of road dangers. The drivers judged 30 traffic situations in each session. The arbitrary sounds resulted in longer response times and degraded response accuracy compared to the other two conditions. A one-way ANOVA revealed significant differences in response time $F(2,34)=27.56, p<0.01$ and accuracy $F(2,34)=56.19$, $p<0.01$. A Tukey's HSD test showed a significant difference between the arbitrary condition and the two other conditions $(p=0.01)$. Significant effects were also found both in terms of identification of road dangers $F(2,34)=69.99, p<0.01$ and position of road dangers $F(2,34)=16.77, p<0.05$. A Tukey's HSD test found that the arbitrary sounds resulted in more errors both in terms of identity and position of road dangers $(p=0.01)$.

\subsection{Subjective ratings}

The subjective ratings of pleasantness and cognitive effort are presented in Table 5. The arbitrary sounds were rated more annoying and more challenging to interpret while simultaneously performing the driving task compared to the other two sound sets. A significant effect was found for interpretation $F(2,34)=15.32, p<0.01$ and annoyance $F(2,34)=7.47$. The post-hoc analysis reported significant effects between the arbitrary sounds and the other conditions $(p=0.01)$.

Table 4 Mean accuracy of response in terms of identity and position of road users

\begin{tabular}{|l|l|l|}
\hline & Identity \% (SD) & Position \% (SD) \\
\hline meaningful & $2.4(5.6)$ & $12.8(9.8)$ \\
\hline arbitrary & $26.7(12.2)$ & $23.9(15.0)$ \\
\hline verbal & $2.6(5.8)$ & $10.9(7.8)$ \\
\hline
\end{tabular}

Table 5 Mean subjective ratings of pleasantness and cognitive effort

\begin{tabular}{|l|c|c|}
\hline & Pleasantness (SD) & Cognitive effort (SD) \\
\hline meaningful & $3.45(2.09)$ & $5.61(1.97)$ \\
\hline arbitrary & $5.39(2.83)$ & $7.39(1.78)$ \\
\hline verbal & $3.56(1.76)$ & $5.50(2.15)$ \\
\hline
\end{tabular}

\section{Discussion}

The purpose of the study was to evaluate learnability, cognitive demand and pleasantness of auditory signs designed to support drivers' traffic SA. Sounds assumed to have a natural meaning in the driving context were compared with arbitrary sounds and verbal signs. In the interview carried out at the end of each trial, a majority of the drivers reported that they tried to make the arbitrary sounds meaningful by finding intuitive similarities and forming associations between the signs and the road users. For instance, one subject stated, 'the musical motive representing the big truck was the easiest one to recognise since the cello is playing in a low register'. This association definitely makes sense. Large objects vibrate more slowly than small objects and thus produce lower tones. An instrument playing in a low register could therefore signify a large object moving. Another driver said that he tried hard to establish an association between the sound of a trumpet and a child playing the trumpet. However, most drivers reported that they failed to establish durable and useful associations between the arbitrary sounds and the traffic events. Even though they spent considerable more time and trials trying to learn the meaning of the sounds, their judgment performance was degraded in this condition compared to the other two conditions. Significant effects were found both in terms of response time and accuracy of response. In fact, none of the 18 truck drivers performed better when interpreting the arbitrary sounds compared to the other two conditions.

Taken together, the results of the experiment support the hypothesis that sounds that are meaningful in the driving context require considerable less cognitive resources compared to sounds arbitrarily mapped to traffic information. This effect was found even though drivers spent more time and trials learning the arbitrary sounds. The lack of meaning was apparently not compensated by the longer learning sessions. Many sounds we encounter in user environments are arbitrary and meaningless sounds. The results of this study emphasise the use of sounds that have a natural meaning in the driving context and are easily associated with their intended meaning. The study also demonstrates that brief sounds imitating road users may be appropriate when designing especially for traffic SA.

During the trials the drivers were required to respond to the sounds as fast as possible so that it would not affect driving performance more than absolutely necessary. The 
drivers were told they were judged primarily on the basis of their driving performance. But sometimes the arbitrary sounds resulted in exceptionally long response times (10$15 \mathrm{~s})$. This happened for three subjects (17\%) and represented $1.5 \%$ of the measurements. In these situations, the drivers were not able to find a solution within a reasonable timeframe. Despite the time pressure, they remained motionless staring at the touch screen for long periods, completely losing focus on the road. It seems like the arbitrary sounds contributed to a severe level of attentional narrowing or even cognitive overload for some drivers. It can be argued that the attentional narrowing was intensified by stress induced by the time pressure [30]. The behaviour was very inappropriate since the drivers were not able to focus visually on the screen and on the road scene simultaneously. In a real driving situation, longer periods of attentional narrowing induced by interface elements could significantly impact on drivers' ability to perceive and process other driving-related information.

The drivers performed equally fast and accurate when interpreting meaningful non-verbal signs as they did when interpreting short verbal signs. This motivates a more extensive comparison between these two types of sounds. They both have potential advantages and disadvantages. Speech is known to be sensitive to other verbal communication or background noise. However, a modern truck cab is a relatively controlled sound environment. Some newer models can even mute sounds (radio, telephone signals, etc.) that are not considered appropriate in the particular situation. Vilimek and Hempel [21] found that long verbal messages $(>3.5 \mathrm{~s})$ could have a negative impact on short-term memory. But if keywords can be used, verbal messages seems promising.

A strong argument to develop non-verbal signs is the potential to find more universal signs that are not language dependent. Also, recent research on verbal communication has promoted the importance of voice adaption when designing for in-vehicle systems [31]. Voice familiarity, gender, age and emotional tone may have considerable impact on both system driver attitude and driving performance. No particular type of voice seems to fit all drivers, which in turn can make development of speechbased displays challenging.

One general issue with meaningful non-verbal sounds is that they can be hard to find. Some objects in a traffic scene do not even produce sound. Producing comprehensible speech-based messages may be easier.

A general issue in audio interface design is how to find sounds that will not be annoying. Annoyance may be a desirable quality in some very urgent situations. In other words, in most everyday situations, it is important to find signals that are pleasant to listen to. In the present study, the participants rated perceived annoyance at the end of each experimental condition. That is, the driver rated the arbitrary assigned sounds more annoying than the more meaningful sounds. The results give us some indication of how arbitrary sounds implemented in vehicles can have a negative impact on user satisfaction. McKeown [15] also reported low scores of pleasantness for abstract sounds compared to other verbal and non-verbal signs.

One potential criticism of the study is that the laboratory set-up is not really comparable to realistic driving. The lane change test requires the driver to focus on the forward road scene. However, it does not fully represent the dynamic task of driving. Also, in the study the drivers' interpreted 90 auditory signs in about $1 \mathrm{~h}$ of driving. This high frequency of sounds would have promoted a higher attentiveness than is typical in routine driving.

\section{Future work}

The concept of using sound as a primary channel for trafficrelated information need to be further evaluated. Future experiments should examine driver's behaviour and effects on driving that can be directly related to safety. Also, previous work has shown how complexity of the traffic environment can significantly affect workload, both for experienced and inexperienced drivers [32]. Future evaluations should address the efficiency of auditory signs in traffic situations with different levels of complexity. Finally, we still know little about how auditory displays using auditory signs can disturb and confuse drivers during real driving. Thus, it would be very interesting to validate the result of the present study in a real-world setting. Such a study would give us a better understanding of how informative auditory displays may support and confuse experienced drivers during real driving.

\section{Acknowledgments}

Scania CV AB and The Swedish Governmental Agency for Innovation Systems (Vinnova) financed this work. A special thanks to Stefan Lindberg at the Interactive Institute for helping us with the sound design, and to Robert Friberg and Josefin Nilsson at Scania CV AB for support and inspiration.

\section{References}

[1] KIRCHER A., LINDER A., NYGÅRDHS S., VADEBY A.: 'Intelligenta transportsystem, ITS, i passagerarbilar och metoder för utvärdering av dess inverkan på trafiksäkerheten' (Swedish National Road and Transport Research Institute, Linköping, Sweden, December 2007, 1st edn.)

[2] ALM H., NILSSON L.: 'The effects of a mobile telephone task on driver behavior in a car following situation', Accident Anal. Prev., 1995, 27, (5), pp. 707-715 
[3] ENGSTRÖM J., JOHANSSON E., ÖSTLUND J.: 'Effects of visual and cognitive load in simulated motorway driving', Transp. Res. Part F, 2005, 8, (2), pp. 97-120

[4] StRayer D.L., DReWS F.A.: 'Cell-phone-induced driver distraction', Curr. Dir. Psychol. Sci., 2007, 16, (3), pp. 128-131

[5] NeAlE V.L., DinguS T.A., KLAUER S.G., SUDWEEKS J., GOODMAN M.: 'An overview of the 100-car naturalistic study and findings'. National Highway Traffic Safety Administration, Washington, DC, USA, 2005, Paper No. 05-400

[6] RECARTE M.A., NUNES L.M.: 'Effects of verbal and spatialimagery task on eye fixations while driving', J. Exp. Psychol. Appl., 2000, 6, (1), pp. 31-43

[7] PATTEN C., KIRCHER A., ÖSTLUND J., NILSSON L.: 'Using mobile phones: cognitive workload and attention resource allocation', Accident Anal. Prev., 2004, 36, (3), pp. 341-350

[8] MCCARLEY J.S., VAIS M.J., PRINGLE H., KRAMER A.F., IRWIN D.E., StRAYER D.L.: 'Conversation disrupts change detection in complex traffic scenes', Hum. Factors, 2004, 46, (3), pp. $424-436$

[9] RICHARD C.M., WRIGHT R.D., EE C.: 'Effect of a concurrent auditory task on visual search performance in a drivingrelated image-flicker task', Hum. Factors, 2002, 44, (1), pp. $108-119$

[10] KASS S.J., COLE K., LEGAN S.: 'The role of situation awareness in accident prevention' in DE SMET A. (ED.): 'Transportation accident analysis and prevention,' (Nova Science Publishers, Inc., 2008, 1st edn.), pp. 107-122

[11] ENDSLEY M.R.: 'Design and evaluation for situation awareness enhancement'. Proc. Human Factors Society 32nd Annual Meeting, Santa Monica, USA, 1988

[12] FUNG C.P., CHANG S.H., HWANG J.R., HSU C.C., CHOU W.J., CHANG K.K.: 'The study on the influence of auditory warning systems on driving performance using a driving simulator'. Institute of Transportation, Taiwan, 2007, Paper no. 07-0170

[13] HO C., SPENCE C.: 'Assessing the effectiveness of various auditory cues in capturing a driver's visual attention', J. Exp. Psychol. Appl., 2005, 11, (3), pp. 157-174

[14] HO C., SPENCE C., TAN H.z.: 'Warning signals go multisensory'. Proc. HCl Int. 2005, Las Vegas, USA, 2005

[15] MCKEOWN D.: 'Candidates for within-vehicle auditory displays'. Proc. Int. Conf. Auditory Display, Limerick, Ireland, 2005

[16] GAVER W.W.: 'The SonicFinder, a prototype interface that uses auditory icons', Hum. Comput. Interact., 1989, 1, pp. 67-94
[17] WALKER B.N., NANCE A., LINDSAY J.: 'Spearcons: speech based earcons improve navigation performance in auditory menus'. Proc. Int. Conf. Auditory Display, London, UK, 2006

[18] ULFVengren P.: 'Design of natural warning sounds'. Proc. Int. Conf. Auditory Display, Montreal, Canada, 2007

[19] Blattner M., SumikaWA D., Greenberg R.: 'Earcons and icons: their structure and common design principles', Hum. Comput. Interact, 1989, 4, (1), pp. 11-44

[20] GAVER W.W.: 'Auditory icons: using sound in computer interfaces', Hum. Comput. Interact., 1986, 2, (2), pp. $167-177$

[21] VILIMEK R., HEMPEL T.: 'Effects of speech and non-speech sounds on short-term memory'. Proc. Int. Conf. Auditory Display, Limerick, Ireland, 2005

[22] BUSSEMAKERS M.P., DE HAAN A.: 'When it sounds like a duck and looks like a dog... auditory icons vs. earcons in multimedia environments'. Proc. Int. Conf. Auditory Display, Atlanta, USA, 2000

[23] DINGLER T., LINDSAY J., WALKER B.: 'Learnability of sound cues for environmental features: auditory icons, earcons, spearcons and speech'. Proc. Int. Conf. Auditory Display, Paris, France, 2008

[24] LEUNG Y.K., SMITH S., PARKER S., MARTIN R.: 'Learning and retention of auditory warnings'. Proc. Int. Conf. Auditory Display, Paolo Alto, USA, 1997

[25] LeMmenS P.M.C., BUSSEMAKERS M.P., DE haAN A.: 'Effects of auditory icons and earcons on visual categorization: the bigger picture'. Proc. Int. Conf. Auditory Display, Espoo, Finland, 2001

[26] STEPHAN K.L., SMITH S.E., PARKER S.P.A., MARTIN R.L., MCANALLY K.I.: 'Auditory warnings in the cockpit: an evaluation of potential sound types'. Innovation and consolidation in aviation: selected contributions to the Australian Aviation Psychology Symp. 2000, 2003

[27] BREWSTER S., WRIGHT P., EDWARDS A.: 'A detailed investigation into the effectiveness of earcons'. Proc. Int. Conf. Auditory Display, Santa Fe, USA, 1992

[28] MUSTONEN M.S.: 'A review-based conceptual analysis of auditory signs and their design'. Proc. Int. Conf. Auditory Display, Paris, France, 2008

[29] CHEN F., JARLENGRIP J.: 'Listen! there are other road users close to you - improve the traffic awareness of truck drivers,' in STEPHANIDIS C. (ED.): 'Universal access in human computer interaction, ambient interaction' (Springer, 2007, 1st edn.), pp. 323-329 
[30] KOWALSKI-TRAKOFLER K.M., SCHARF T., VAUGHT C.: 'Judgement and decision making under stress: an overview for emergency managers', Emer. Manage., 2003, 1, (3), pp. 278-289

[31] JONSSON I.M.: 'Social and emotional characteristics of speech-based in-vehicle information systems: impact on attitude and driving behaviour'. PhD thesis, Linköping University, 2009

[32] PATTEN C., KIRCHER A., ÖSTLUND J., NILSSON L., SVENSON O.: 'Driver experience and cognitive workload in different traffic environments', Accident Anal. Prev., 2006, 38, (5), pp. 887-894 